\title{
The Influence of $\boldsymbol{\gamma}$-Irradiation on Structuration in Solutions of BNR and Properties of Films
}

\author{
Shiraz M. Mammadov ${ }^{1}$, Adil A. Garibov ${ }^{1}$, Oktay A. Akparov², Sehrana A. Rzayeva, \\ Akif K. Salehov ${ }^{1}$, Sahile S. Ahmadova ${ }^{1}$ \\ ${ }^{1}$ Institute of Radiation Problems of ANAS, Baku, Azerbaijan \\ ${ }^{2}$ Baku State University, Baku, Azerbaijan \\ Email: siraz_mm55@mail.ru
}

Received January 25, 2012; revised February 28, 2012; accepted March 30, 2012

\begin{abstract}
Studied influence of $\gamma$-irradiation on stucturation in butadiene nitrile rubber solution and films properties studied also. Structuration in solutions has defined by rheological method with rotation viscometer. Shown influence of solvent nature (methyl ethyl ketene: toluene) to strength properties and structures of films obtained from this solvents. It has been found that during irradiation of solvent, films and butadiene nitrile rubber solution characteristic viscosity $\eta$ increased, increasing of Huggins constant ( $\left.\mathrm{K}^{\prime}\right)$ observed as well. Changing of spatial mesh of polymers occurs at the cost of changed size of macromolecule balls. Shown, that in the issue of crossing the properties including thermo dynamical features of films and butadiene nitrile rubber solution has changed.
\end{abstract}

Keywords: Cross-Linking; Solvent; Rheology; Relaxation; Spectrometer; Radiation; Viscosity; Irradiation

\section{Introduction}

Physical-mechanical properties of polymeric materials along with the chemical nature of macromolecules in large extent are determined by the structure which is formed under the various factors accompanying the process of recycling. It has been shown in several investigations that structural-mechanical properties of solutions [1-5], and generated films from them [6] can be vary over by combining solvents and precipitants in the wide range. Maximum physical-mechanical properties meet the formation of ordered structures characterized by the highest perfection of relaxation processes $[7,8]$. Consequently, the structure that determined the mechanical properties of the material originates in the solution and in the certain degree captured in the transition to the solid state.

The research of the influence of the various factors $(\gamma$-irradiation) in the structuration process defining the complex of the mechanical properties of the materials is the actual in the light. It was done on base of the example of BNR solutions at current research.

\section{Method and Object of Research}

The object of study was BNR, (butadiene-nitrile-rubberBNR-40). It means by the joint coagulation of butadienenitrile latex (with a cohesive acrylonitrile $37 \%$ - 40\%) as a result of studies by the IR-spectroscopy method in the part of butadiene under temperature $300 \mathrm{~K}$ the polymerization of the isomeric compounds of double bonds in the investigated polymer is 1,2 -isomer $14.3 \%, 1,4$-isomer $14.8 \%$ and trans 1,4 -isomers $70.9 \%$.

The structure formation in solutions was researched by rheological method in rotary viscometer range $4.3 \times 10^{-3}$ $-480 \mathrm{c}^{-1}$.

The process of the structuration was judged by the magnitude of the maximum $\dot{\eta}_{\mathrm{o}}$ and minimum $\dot{\eta}_{\infty}$ of constant viscosity, the nature of the dependence of viscosity on the shear tension $\dot{\eta}=f(\mathrm{p})$, the limiting magnitude of the shear tension $\mathrm{P}_{\mathrm{K}}$. The structure and properties of the films was identified by the kinetics of the isothermal increase of strength after fracture.

The quality of solvent was evaluated by the capillary viscometer as viscometer type Ubbelohde with capillary diameter $0.60 \mathrm{~mm}$.

The optical properties of system were investigated with spectrometer. For irradiation of BNR solutions and films with shown compounds were placed in vials (ampoules) made of glass like "Ray". Irradiation of the sealed ampoule was performed $\gamma$-rays under power $\mathrm{Co}^{60}$ at room temperature $4.9 \mathrm{D} / \mathrm{c}$. The absorbed dose was 50 $\mathrm{kGy}$.

The mechanical properties of the films was determined by a standard method at the strain-rate $20 \mathrm{~mm} / \mathrm{min}$ on tensile testing machine "Instron".

The result of measurement of mechanical properties: 
tensile strength $\sigma$ and comparative elongation in the time of tear of film $\varepsilon$ are presented in Table $\mathbf{1}$.

\section{Results and Discussion}

It is obvious that, the dependence of $\sigma$ from the solvent compound has extreme nature: maximum strength for films, formed from system MEK:Toluene $=1: 0.5$. Also the films have enough high strength, obtained from a mixture $1: 1$. The further increase of toluene content leads to a decrease of strength of the films, however, in that case $\sigma$ is still much higher than $\sigma$ films which formed from MEK.

In Table 1 presented the result of researches of dilute solutions by the capillary viscometry method. It is obvious that, with the increasing concentration of toluene up to $30 \%$ characteristic viscosity [' ${ }^{\prime}$ ] reduced slightly, but viscometric constant Huggins $K^{\prime}$ increases (i.e. the quality of solvent decreases), herewith the size of the macromolecule coils do not change practically. It is possible only during confirmatory rebuilding of BNR macromolecule consisting of units of butadiene and acrylonitrile.

The further increase of toluene proportion in the mixture leads to low decrease of [ $\left.\eta^{\prime}\right]$ and increase of $\mathrm{K}^{\prime}$ i.e. the quality of solvent degrades the size of the macromolecule coils decrease. In the compound range, corresponding to the high content of toluene a sharp decrease of BNR solvability and the transition of the system to two-phase state is observed.

The spectrophotometric study of BNR solutions in MEK shows that even at $1 \%$ concentration of polymer in solution is observed deviation of the optical density, i.e. the macromolecules present in solution as associates, which is caused, apparently, the ability to form strong carbon bonds, as well as the tendency of the polymer to crystallization. The deterioration of thermodynamic quality of the solvent shifts the threshold of aggregation in low concentration, as in MEK solution: Toluene compound $1: 3$ and $1: 4$ is the critical concentration of 0.5 and $0.25 \%$ respectively. Thus, the observed extreme changes of mechanical properties of BNR films are the result of

Table 1. Influence of the solvent compounds on properties of films and BNR solutions.

\begin{tabular}{ccccc}
\hline $\begin{array}{c}\text { Solvent compounds } \\
\text { MEK:Toluene }\end{array}$ & {$\left[\dot{\eta}^{\prime}\right]$} & $\mathrm{K}^{\prime}$ & $\sigma, \mathrm{Pa}$ & $\varepsilon, \%$ \\
\hline $1: 0$ & 0.665 & 0.47 & 0.10 & 260 \\
$1: 0.5$ & 0.66 & 0.50 & 2.60 & 780 \\
$1: 1$ & 0.69 & 0.52 & 2.10 & 800 \\
$1: 2$ & 0.55 & 0.60 & 1.40 & 740 \\
$1: 3$ & 0.59 & 0.30 & - & - \\
$1: 4$ & 0.53 & 0.28 & - & - \\
\hline
\end{tabular}

deterioration of thermodynamic quality of solvent.

The results of radiation chemical researches of structure of films and BNR solutions formed from the thermodynamic quality solution are shown in Table 2 .

It is obvious that during irradiation of the same solvent compounds, films and BNR solutions characteristic viscosity [ $\left.\eta^{\prime}\right]$ increases, the growth of Huggins constant $K^{\prime}$ is observed, the parameter of the spatial grid of the polymer changes due to alteration in the size of the coils of the macromolecules, the three-dimensional structure of the transmission to the cross-linking forms, and the physicalmechanical properties of films and BNR solutions including the thermodynamic quality solvent changes.

In concentrated solutions area the influence of thermodynamic quality of the solvent on the structural state of the system observes more sharply (Figure 1).

The increase of the toluene leads not only the increase of the absolute values of viscosity, but also the increase

Table 2. Influence of $\gamma$-irradiation on the solvent compounds, properties of film and solutions BNR $(D=50 \mathrm{kGy})$.

\begin{tabular}{ccccc}
\hline $\begin{array}{c}\text { Solvent compounds } \\
\text { MEK:Toluene }\end{array}$ & {$[\dot{\eta}]$} & $\mathrm{K}^{\prime}$ & $\sigma, \mathrm{Pa}$ & $\varepsilon, \%$ \\
\hline $1: 0$ & 0.87 & 0.58 & 1.14 & 350 \\
$1: 0.5$ & 0.91 & 0.62 & 3.60 & 670 \\
$1: 1$ & 0.86 & 0.70 & 3.30 & 690 \\
$1: 2$ & 1.0 & 1.1 & 2.30 & 650 \\
$1: 3$ & 1.12 & 0.87 & 1.45 & 800 \\
$1: 4$ & 0.61 & 1.30 & 1.30 & 850 \\
\hline
\end{tabular}

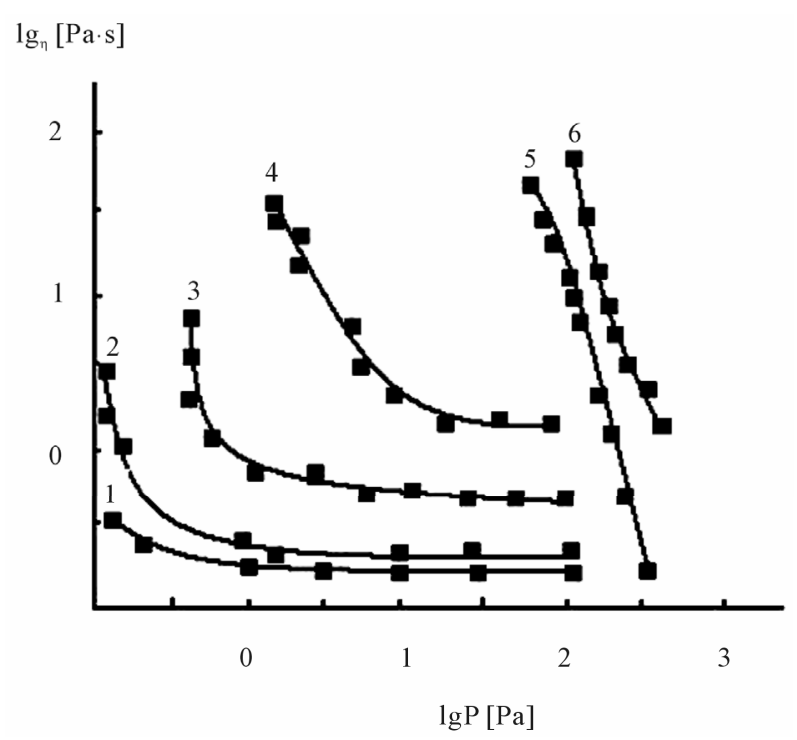

Figure 1. Influence of thermodynamic quality of the solvent on the rheological properties of $10 \%$ BNR solutions. MEK:T $=1: 0(1), 1: 0.5(2), 1: 1.45(3), 1: 1.8(4), 1: 2.21(5)$, and 1:4.28 (6). 
of viscosity anomaly, i.e. the increase of structuring of the system. At sufficient high substance of toluene (curves 5,6 ) along with the sharp increase of viscosity changes the dependency character $\dot{\eta}=f(\mathrm{p})$ : curve is mixed in the zone of high shear tension, but the transition from $\dot{\eta}_{\mathrm{o}}$ into $\dot{\eta}_{\infty}$ occurs rapidly in the narrow range of high shear tension.

Draws attention the fact that in this case the value $\dot{\eta}_{\infty}$ is in lower value obtained for the previous systems (curves 1 - 4). It is due to phase change system.

The phase diagrams of the ternary BNR-MEK-Toluene was constructed by the method of isothermal titration BNR in MEK precipitator (toluene). Unfortunately, to establish the compound of the precipitating phase failed.

Thus, deterioration of thermodynamic quality of solvents alters the character of the structure of macromolecules, i.e. the resulting spatial patterns: in case of a solution this spatial grid is formed by the fluctuating nature, but in case of dispersion clutch assemblies the new phase of macromolecules BNR.

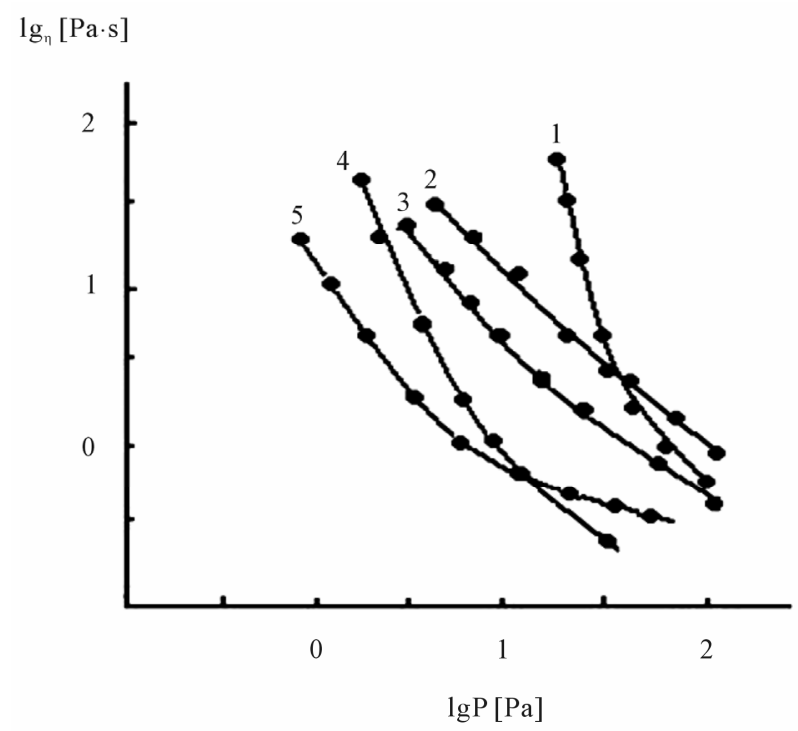

Figure 2. Influence of radiation on the rheological properties of $15 \%$ BNR solutions in the solvent of different thermodynamic quality.
Due to the fact that the deterioration of thermodynamic quality of the solvent leads to increased interaction of polymers, the strength of inter-structural contacts increases, the total destruction of the structure occurs at higher shear stresses.

In Figure 2 the data were shown characterizing the influence of irradiation dose on the strength of inter-structural contacts. It is obvious that in case of the dose increasing of the solution leads to a decrease $\dot{\eta}_{\infty}$, but structuration is enhanced in the area of the low loads with increasing doses. In the two-phases systems the dose increasing leads to a decrease of strength of inter-structural contacts (Figure 2, curves 1,2), so that the structural viscosity decreases, but $\dot{\eta}_{\infty}$ increases.

If the system is in a state close to the bundle, in this case the temperature rise reduces as a structural and effective viscosity of the solution. Curves (Figure 2) corresponding to the system at fifth represents the mixed case, when dose increasing of irradiation leads to a transition from a two-phase in a single-phase region.

\section{REFERENCES}

[1] S. P. Pankov, "Ravnovesiya faz v Sisteme PolimerRastvoritel," M.: Chemistry, 1981, p. 234.

[2] S. P. Pankov, "Studneobraznoe Sostoyanie Polimerov," M.: Chemistry, 1984, p. 237.

[3] V. G. Kulichikhin, A. Y. Malkin and S. P. Pankov, Visokomol. Soed. A, Vol. 24A, No. 11, 1982, p. 2291.

[4] Mandelkern, "Kristalizasiya Polimerov," Frenkel S.Y. L.: Chemistry, 1976, p. 296.

[5] M. E. Kurbanaliyev, I. K. Dustov and A. Y. Malkin, Visokomolek. Soed. A, Vol. 24, No. 11, 1984, p. 2291.

[6] P. I. Zubov, A. I. Zemcev and L. L. Sukhareva, Kolloid. Zhurn., Vol. 38, No. 4, 1986, p. 66.

[7] S. A. Tashmuhamedov, S. A. Azizov, B. U. Sagdullayev and Z. G. Sagdiyeva, Visokomolek. Soed. A, Vol. 20, No. 1, 1988, p. 1180 .

[8] I. Prigozhin and R. Dephey, Khimicheskaya Termodinamika. M., Khimiya, 1978, p. 306. 